\title{
RAIDD is required for apoptosis of PC12 cells and sympathetic neurons induced by trophic factor withdrawal
}

\author{
Q Wang ${ }^{1,4}$, M Maniati $^{2,4}$, O Jabado ${ }^{1}$, M Pavlaki $^{2}$, CM Troy ${ }^{1,3}$, \\ LA Greene ${ }^{3}$ and L Stefanis ${ }^{\star, 1,2,3}$ \\ ${ }^{1}$ Department of Neurology, Columbia University, New York, USA \\ 2 Neurobiology Laboratory, Foundation of Biomedical Research of the Academy \\ of Athens, Athens, Greece \\ ${ }^{3}$ Department of Pathology, Columbia University, New York, USA \\ ${ }^{4}$ These authors have contributed equally to this work \\ * Corresponding author: L Stefanis, Neurobiology Laboratory, Foundation for \\ Biomedical Research of the Academy of Athens, Soranou Efesiou 4, Athens \\ 11527, Greece. Tel: + 30210-6597214 or 30-210-6597498; \\ Fax: +30-210-6597545; E-mail: Istefanis@ bioacademy.gr
}

Received 03.1.05; revised 06.4.05; accepted 12.4.05; published online 10.6.05 Edited by $\mathrm{G}$ Salvesen

\section{Abstract}

Caspase 2 has been implicated in trophic deprivation-induced neuronal death. We have shown that overexpression of the caspase 2-binding protein RAIDD induces neuronal apoptosis, acting synergistically with trophic deprivation. Currently, we examine the role of endogenous RAIDD in apoptosis of PC12 cells and sympathetic neurons. Expression of a truncated caspase recruitment domain-only form of caspase 2, which presumably disrupts the RAIDD interaction with endogenous caspase 2, attenuated trophic deprivationinduced apoptosis. Furthermore, downregulation of RAIDD by small interfering RNA led to inhibition of trophic deprivation-induced death, whereas death induced by DNA damage, which is not caspase 2-mediated, was not inhibited. Therefore, RAIDD, likely through interaction with caspase 2 , is involved in trophic deprivation-induced neuronal apoptosis. This is the first demonstration of the involvement of RAIDD in apoptosis, and provides further support for the idea that apoptotic pathways in the same system may differ depending on the initiating stimulus.

Cell Death and Differentiation (2006) 13, 75-83.

doi:10.1038/sj.cdd.4401690; published online 10 June 2005

Keywords: adaptor protein; PC12 cells; caspase 2; sympathetic neuron; siRNA

Abbreviations: CARD, caspase recruitment domain; siRNA, small interfering RNA; FRAIDD, Flag-tagged rat RAIDD; FhRAIDD, Flag-tagged human RAIDD; ctl, control; scr, scrambled; Ab, antibody; $\mathrm{C} 2$, caspase 2; $\mathrm{HMW}$, high molecular weight

\section{Introduction}

Apoptosis generally occurs through an 'intrinsic' or 'stress', and an 'extrinsic' or 'death receptor' pathway. In both cases, there is involvement of caspases. Caspases are intracellular cysteine aspartases that are activated in cell death and inflammatory pathways. ${ }^{1,2}$ The 13 known vertebrate members of this protease family can be categorized into different groups, depending on their relative homology, their substrate and inhibitor specificity, their involvement in apoptosis or inflammation, their early or late position in apoptotic pathways, and the presence or absence of large $\mathrm{N}$-terminal prodomains. ${ }^{2}$ Most of the caspases fall neatly within these categories, but the situation for caspase 2 is more complex. Caspase 2 is the mammalian caspase most closely related to the $C$. elegans ced3 caspase. ${ }^{3}$ It has a large prodomain and high homology with caspases 1 and 9, which are 'apical' or 'initiator' caspases. On the other hand, it has a synthetic substrate specificity that is similar to that of the 'effector' caspase 3 and 7 , and has been shown in many systems to be processed by such effector caspases, situating it in a downstream position in apoptotic pathways. ${ }^{2,4}$

A variety of studies support the idea that caspase 2 may be a 'master regulator' caspase in the intrinsic pathway. ${ }^{5-10}$ Furthermore, the possibility has been raised that it may act independently of the caspase 9-related apoptosome, which may serve only to amplify the signal, but not to determine life or death. ${ }^{5,11}$ We have shown that caspase 2 is involved in apoptosis induced by trophic deprivation in PC12 cells and sympathetic neurons. ${ }^{12,13}$ The relevance of such data were questioned when caspase 2 null mice were reported to have very few defects in apoptosis and, in particular, no defect in NGF deprivation-induced death of cultured sympathetic neurons. ${ }^{14,15}$ We have found that a major cause for this discrepancy is the compensatory upregulation in caspase 2 null neurons of the caspase 9 pathway, which is normally held in check by an inhibitor of apoptosis family member, XIAP. ${ }^{16}$ Recent findings from others, using a dominant-negative strategy, have confirmed the requirement for caspase 2 in this system. ${ }^{17}$

Caspases are generally activated either through processing at aspartate residues or through homo- or hetero-oligomerization. $^{2}$ The latter scenario generally applies to long prodomain-containing caspases, such as caspase 2. Caspase 2 contains in its $\mathrm{N}$-terminal domain a caspase recruitment domain (CARD), which interacts with the CARD of at least two other proteins, RAIDD/CRADD and ARC. ${ }^{18-21}$ However, binding of these adaptors to caspase 2 has not been conclusively shown in a cellular context following a physiological death stimulus. Therefore, the manner of caspase 2 activation remains elusive, and the role of such adaptor proteins is unclear.

We have recently cloned rat RAIDD and have reported that its overexpression in $\mathrm{PC} 12$ cells and neonatal rat sympathetic neurons leads to apoptotic death. RAIDD overexpression acted synergistically with serum deprivation to induce death of PC12 cells. Coexpression of a truncated caspase 2 construct 
containing only the CARD (C2(1-135)) inhibited RAIDDinduced death. ${ }^{22}$ These data raised the possibility that RAIDD may play a role in trophic factor deprivation-induced apoptosis through its interaction with caspase 2 . In this manuscript, we have addressed more directly the role of endogenous RAIDD in trophic factor deprivation-induced apoptosis of PC12 cells and sympathetic neurons.

\section{Results}

\section{RAIDD is present with caspase 2 in the cytosol}

The levels and localization of endogenous RAIDD in PC12 cells and sympathetic neurons at baseline and following trophic deprivation were examined by Western blotting using a RAIDD specific antiserum. We have previously shown that this antiserum recognizes overexpressed rat RAIDD in PC12 cells and sympathetic neurons, both by immunostaining and Western immunoblotting. ${ }^{22}$ In the present experiments, endogenous RAIDD was detected as an approximately $23 \mathrm{kDa}$ band on Western immunoblots of PC12 cell lysates (Figure 1a). The levels of endogenous RAIDD did not change with serum deprivation, whereas caspase 2 was processed, as previously described ${ }^{13}$ (Figure 1a). Similar results were achieved following immunoblotting with two additional commercial antisera directed against RAIDD (from Santa Cruz and US Biochemicals, data not shown).

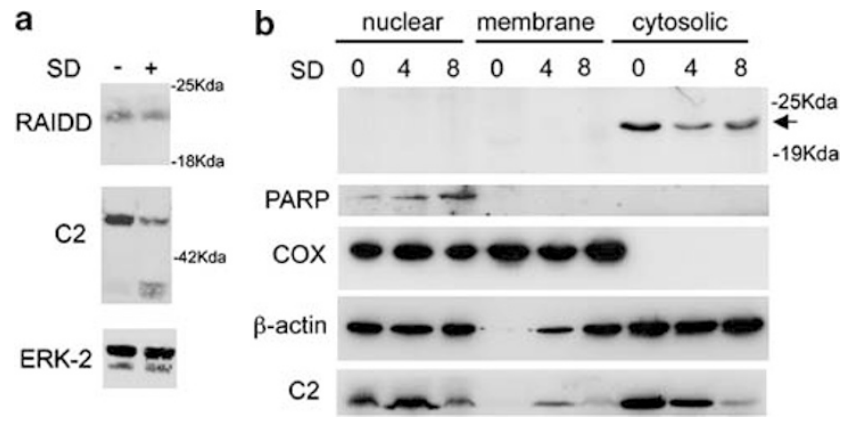

Figure 1 RAIDD colocalizes with caspase 2 in the cytosol and its levels do not change with trophic deprivation. (a) PC12 cells were deprived of serum (SD) or maintained in serum (-), and $5 \mathrm{~h}$ later were lysed. The lysates (50 $\mu \mathrm{g}$ protein) were run on $12 \%$ SDS/PAGE gels and immunoblotted with a RAIDD Ab, or with an N-terminal caspase 2 (C2) Ab. ${ }^{12}$ The RAIDD blot was stripped and reprobed with anti-ERK 2 (bottom panel). Processing of $\mathrm{C} 2$ is apparent, but there is no change in RAIDD levels. Preincubation of the RAIDD antibody with the immunizing peptide resulted in the loss of the $23 \mathrm{kDa}$ band (data not shown). (b) Nuclear, membrane and S-100 cytosolic fractions of 0,4 or $8 \mathrm{~h}$ serum-deprived (SD) PC12 cells were generated. The lysates (100 $\mu \mathrm{g}$ protein) were run on a $12 \%$ SDS/PAGE gel and immunoblotted with a RAIDD Ab (top panel). The $23 \mathrm{kDa}$ RAIDD band is indicated by an arrow. The same lysates were also used for immunoblotting with PARP, COX and $\beta$-actin Abs, as markers for the nuclear, membrane and cytosolic fractions, respectively, and for imunoblotting with the caspase 2 (C2) Ab. Note that while the cytosolic fraction, in which RAIDD and C2 are detected, is free of contaminants from other cellular compartments, the nuclear fractions are not entirely pure, as $\beta$-actin and COX are present. The apparent increase of PARP expression and the decrease of $\mathrm{C} 2$ expression in this compartment at 4 and $8 \mathrm{~h}$ of serum deprivation likely reflect stochastic differences in the purity of the preparation, and were not consistently observed in other experiments
To examine the subcellular localization of RAIDD, we performed fractionations by differential centrifugation followed by Western immunoblotting. RAIDD was detected exclusively in the cytosolic fractions and there was no significant change in levels following serum deprivation (top panel, Figure 1b). A $50 \mathrm{kDa}$ protein corresponding to full-length caspase 2 was present predominantly in the cytosolic fraction, and showed a
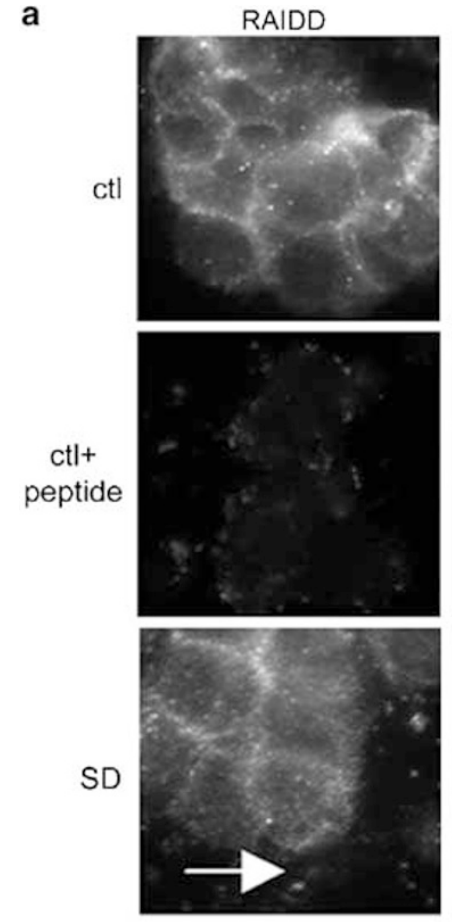

b
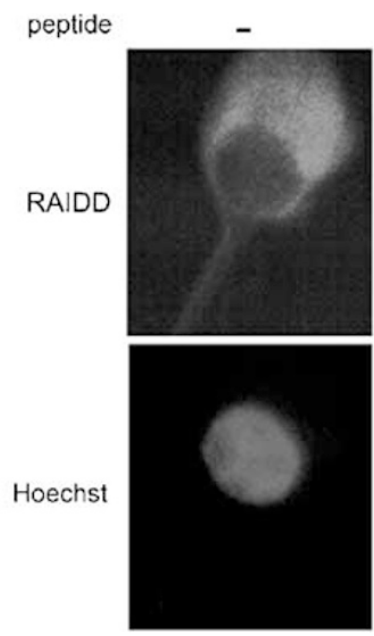
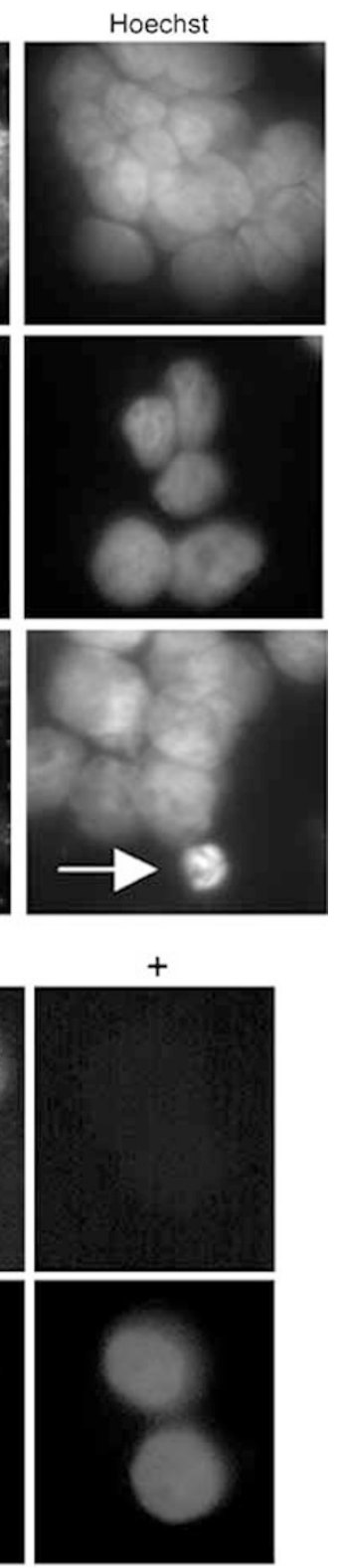

Figure 2 RAIDD localizes to the cytoplasm of PC12 cells and sympathetic neurons and its localization or levels do not change with trophic deprivation. (a) PC12 cells were treated with no additives (ctl) or were serum-deprived (SD). After $12 \mathrm{~h}$, they were fixed and immunostained with a RAIDD $\mathrm{Ab}$ and labeled with Hoechst, with (+ peptide) or without preincubation with a neutralizing peptide. The arrow indicates an apoptotic cell. Images were obtained at $\times 100$ in an inverted fluorescence microscope. (b) Sympathetic neurons were fixed and immunostained with a RAIDD Ab and labeled with Hoechst, with (+ peptide) or without (-peptide) preincubation with a neutralizing peptide. Images were obtained at $\times 40$ in an inverted fluorescence microscope 
diminution of expression in this cellular compartment after serum deprivation (bottom panel). More prolonged exposure of the blot showed that the $37 \mathrm{kDa}$ cleavage product of caspase 2 processing was also present in the cytosolic fraction and increased with serum deprivation (data not shown).

To confirm the above results, we immunostained PC12 cells and sympathetic neurons with the RAIDD antiserum. Immunostaining was cytoplasmic, and was blocked by preincubation with the immunizing peptide (Figure $2 a, b$ ). There was no apparent change in levels or localization following serum deprivation from PC12 cells (Figure 2a) or NGF deprivation from sympathetic neurons (data not shown). Note that the absence of RAIDD staining in an apoptotic cell in Figure $2 \mathrm{a}$ is not a specific phenomenon, as it is observed with most intracellular proteins at these late stages of cellular degeneration.

We conclude that RAIDD is expressed in the cytosol, and that its levels or subcellular distribution do not appreciably change with trophic deprivation. Moreover, as we had previously reported, ${ }^{12}$ the majority of caspase 2 also appears to be localized to the cytosol.

\section{A RAIDD-binding form of the rat caspase 2 CARD protects PC12 cells and sympathetic neurons from trophic factor deprivation-induced apoptotic death}

We have previously reported that a truncated version of rat caspase 2, C2(1-135), containing essentially only the caspase 2 CARD, coimmunoprecipitates with rat RAIDD upon co-overexpression in HEK 293 T cells. ${ }^{22}$ We therefore reasoned that this construct should act as a dominant negative, preventing the putative interaction between endogenous caspase 2 and RAIDD. Thus, if such an interaction is required for activation of caspase 2 , the construct should protect cells from trophic factor deprivation-induced apoptosis. Accordingly, we expressed this Myc-tagged construct in PC12 cells and sympathetic neurons by transient transfection,

Figure 3 Expression of a CARD-only caspase 2 construct attenuates trophic deprivation-induced apoptosis. (a) PC12 cells were transfected with a pCMSEGFP vector encoding myc-tagged C2(1-135). After $36 \mathrm{~h}$, cells were fixed and immunostained with an Myc Ab. Cells were then visualized under a fluorescent microscope. A representative example is shown. Note that the staining is exclusively cytoplasmic. (b) PC12 cells were transfected with pCMS-EGFP vectors encoding Myc-tagged PAI2MT or C2(1-135). After $24 \mathrm{~h}$, the cells were deprived of serum (SD) or maintained in serum (control). After $24 \mathrm{~h}$, the cells were fixed and immunostained with a Myc Ab, followed by Hoechst labeling. PC12 cells expressing C2(1-135) showed significantly less apoptosis compared to those expressing PAI2MT $(P<0.001$, Student's $t$-test). This is representative of four different experiments. (c, d) Sympathetic neuron cultures (5 days old) were transfected with $\mathrm{pCMS}$-EGFP vectors encoding EGFP alone or, in addition, Myc-tagged PAI2MT or C2(1-135). After $24 \mathrm{~h}$, the cultures were either deprived of NGF ( $-N G F)$ or maintained in NGF ( + NGF). After $36 \mathrm{~h}$, the cells were fixed and immunostained with an Myc Ab, followed by Hoechst labeling. Neurons were assessed in individual wells for the presence of apoptotic nuclear features. Results represent $n=4$ for NGF-deprived cultures, and $n=2$ for + NGF cultures. C2(1-135)-expressing neurons showed significantly less apoptosis compared to PAI2MT or EGFP $(P<0.05$, Student's $t$-test). This is representative of three independent experiments. Note in (d) the normal, nonapoptotic nucleus of a C2(1-135)-expressing neuron in the top, and the apoptotic nucleus of a PAI2MT-expressing neuron in the bottom panel and assessed its effects on death induced by trophic factor withdrawal. As controls (ctl's), we used overexpression of EGFP alone, or together with overexpression of an inert form of the serine protease PAI-2 (PAI2MT). C2(1-135) showed an exclusively cytoplasmic form of staining, often in a punctate pattern (Figure 3a). Expression of this construct provided significant protection of PC12 cells from serum withdrawal and of sympathetic neurons from NGF deprivation-induced death (Figure $3 b-d$ ).

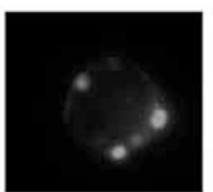

myc

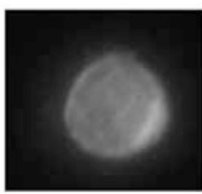

EGFP

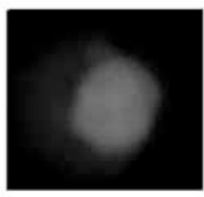

Hoechst
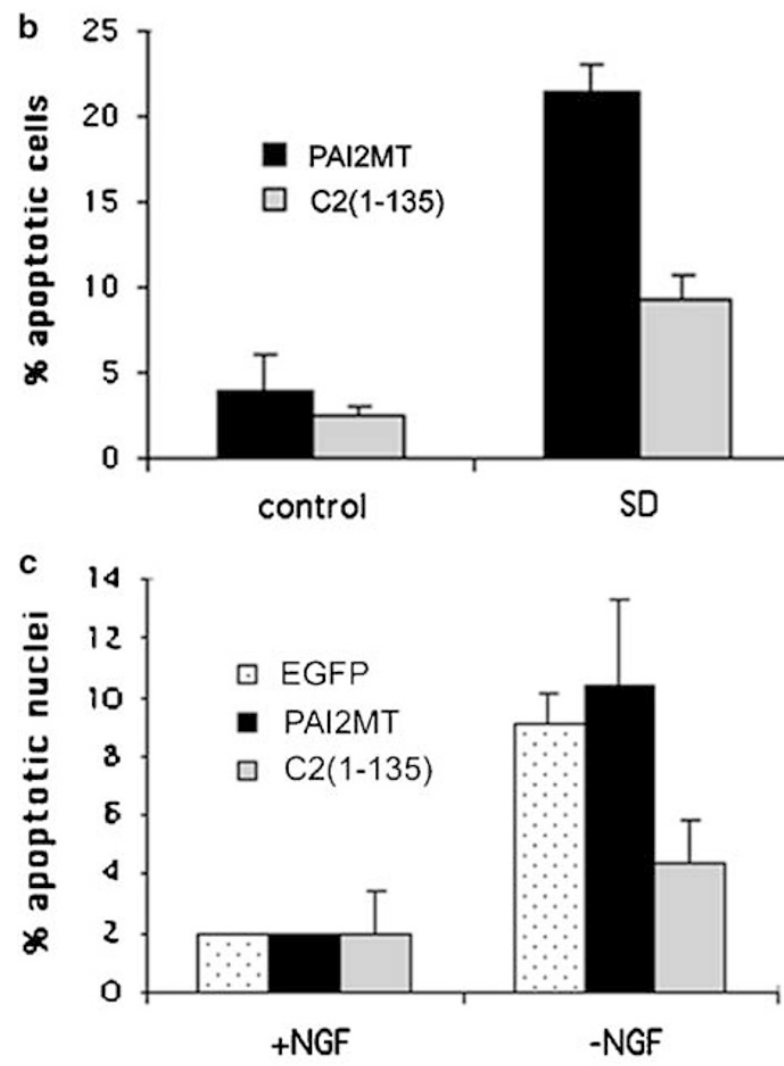

d

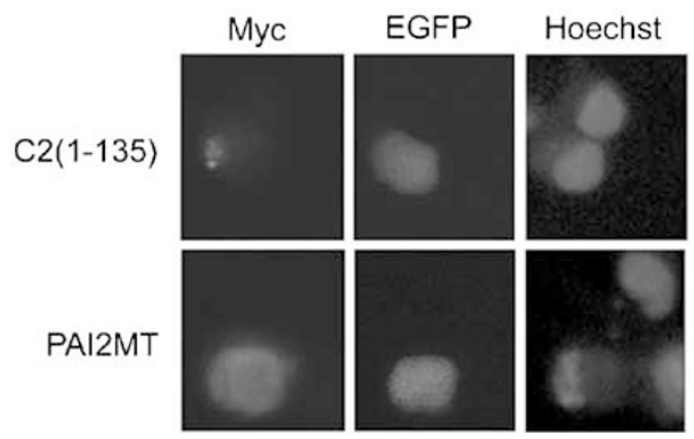




\section{Inhibition of RAIDD expression by specific small interfering RNAs (siRNAs) protects PC12 cells from serum deprivation-induced apoptosis}

To further examine whether RAIDD is involved in trophic factor deprivation-induced apoptosis, we inhibited its expression through the use of siRNA technology. We generated two siRNAs, R1 and R2, against distinct domains of the rat sequence, ${ }^{22}$ and first assessed by immunostaining with the RAIDD-specific antiserum whether they prevented, upon cotransfection, the overexpression of rat RAIDD in PC12 cells (Figure $4 a, b)$. As negative ctls, we used either a ctl siRNA targeted against the transcription factor ATF5, which has no effect on apoptosis in PC12 cells, ${ }^{23}$ or a scrambled (scr) version of R1 that does not target any sequence in the database. Cells overexpressing RAIDD were clearly identified by immunostaining (Figure $4 \mathrm{~b}$, middle column, top row), and thus a quantitative analysis of such cells was possible with each siRNA. When compared to ctl siRNAs, expression of R1 and, to a lesser extent, R2 siRNA inhibited RAIDD overexpression in cells cotransfected with EGFP-RAIDD (Figure $4 a, b)$. Overexpression of Flag-tagged rat RAIDD (FRAIDD ${ }^{22}$ ), as assessed by Flag immunostaining, was also inhibited by R1 and R2 siRNAs (data not shown). This inhibition was specific, because R1 siRNA did not inhibit the expression of overexpressed human RAIDD, which was discordant with this siRNA at a single base pair position (data not shown). To verify these results, we performed Western immunoblots of PC12 cell lysates following overexpression of
FRAIDD, cotransfected with ctl or R1 siRNA. Inhibition of FRAIDD overexpression was readily apparent (right panel) (Figure 4c, arrow heads denote the position of the band corresponding to FRAIDD).

We next examined the effects of siRNA expression on serum deprivation-induced death of PC12 cells, which we have shown in past is dependent on caspase $2 .^{12,13}$ To this end, we cotransfected PC12 cells with EGFP and the various siRNAs, and then deprived them of serum. R1 siRNA significantly inhibited death of EGFP-positive PC12 cells following serum deprivation, when compared to ctl or scr siRNA. R2 siRNA also promoted survival, but to a lesser extent (Figure 5a). To verify that the effect of the siRNA was specific, we took advantage of the fact, mentioned above, that human RAIDD expression was not inhibited by R1siRNA. When human RAIDD was overexpressed together with R1 siRNA, it completely abrogated the protective effect of R1 (Figure 5b). We also assessed whether expression of the siRNAs inhibited serum deprivation-induced apoptosis of EGFP-positive cells, defined by nuclear criteria. R1 and, to a lesser extent, R2 siRNA significantly inhibited such nuclear changes when compared to ctl siRNA (Figure 5c). To examine whether the protective effect of downregulating RAIDD is specific to caspase 2-dependent death, we also exposed PC12 cells to the DNA-damaging agent camptothecin. ${ }^{24}$ Although neuronal death promoted by camptothecin is caspase-dependent, it is not mediated by caspase $2 .^{25}$ In this model, expression of $\mathrm{R} 1$ failed to promote survival (Figure 5d). Therefore, RAIDD does not play a required role

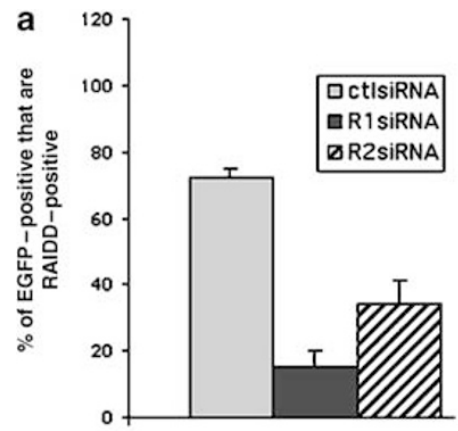

C

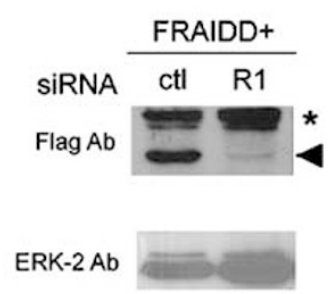

b

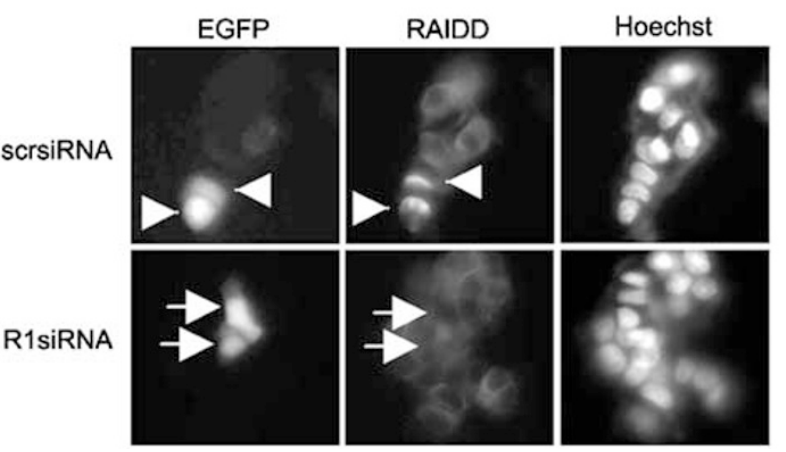

Figure 4 siRNAs targeted against RAIDD suppress RAIDD expression in PC12 cells. (a, b) PC12 cells were transfected with pCMS-EGFP/RAIDD and either control (ctl) or scrambled (scr) R1 or R2 siRNA in 24-well plates. After $48 \mathrm{~h}$, cultures were fixed and immunostained for EGFP and RAIDD. The percentage of EGFP-positive cells that showed intense RAIDD immunostaining was assessed in each setting. Results are representative of four independent experiments. Representative examples are shown in (b). (c) PC12 cells were transfected with ctl or R1 siRNA in combination with pCMS-EGFP/FRAIDD. After $36 \mathrm{~h}$, cells were lysed and lysates were used for Western immunoblotting with a Flag Ab (right panel). The arrow head denotes a $23 \mathrm{kDa}$ band, corresponding to the overexpressed FRAIDD. The asterisk denotes a nonspecific band. The upper part of the blots was used for ERK-2 immunoblotting 
in a death paradigm that is mediated by caspases other than caspase 2.

\section{Inhibition of RAIDD expression by RAIDD siRNA protects sympathetic neurons against NGF deprivation-induced apoptosis}

Next, we investigated whether inhibition of RAIDD expression would have an effect on NGF deprivation-induced death of sympathetic neurons. We first examined whether the siRNAs used in PC12 cells were also effective in downregulating overexpressed rat RAIDD in cultured rat sympathetic
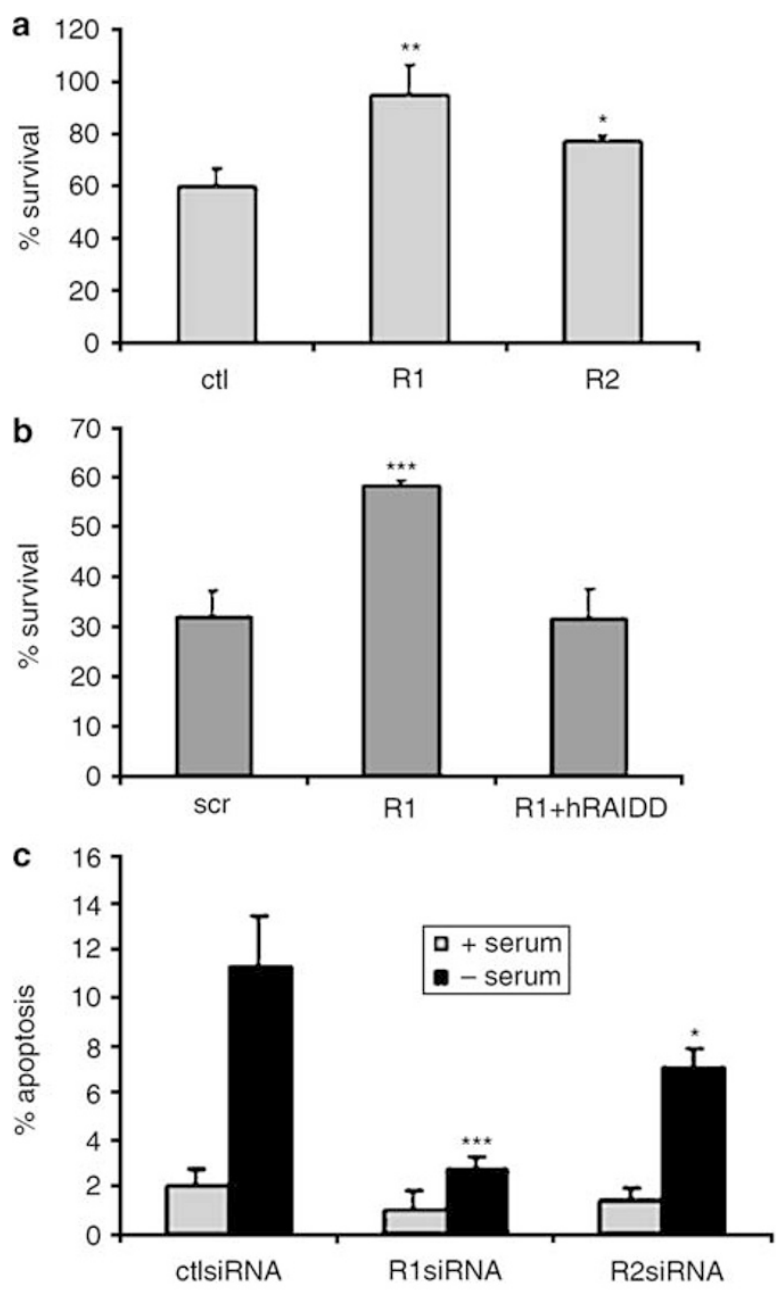

d

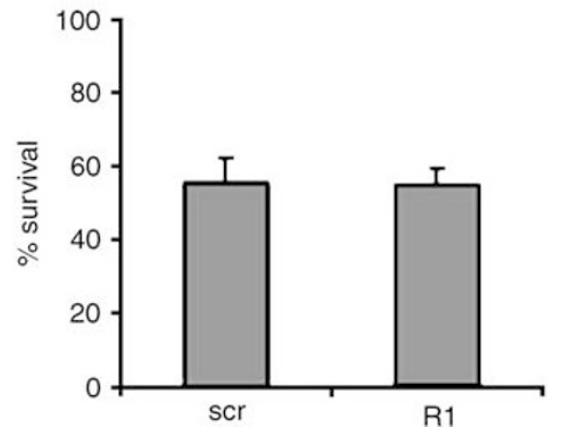

neurons. Using immunostaining, we found that, similar to PC12 cells, R1 and, to a lesser extent, R2 siRNAs indeed downregulated RAIDD (Figure 6a, b). We then went on to assess whether such inhibition of RAIDD altered death of NGF-deprived sympathetic neurons. We found that expression of R1 siRNA almost fully protected sympathetic neurons from death induced by NGF deprivation (Figure 6c). In contrast, R2 siRNA did not have an effect (data not shown). We conclude that inhibition of RAIDD expression prevents death of trophic factor-deprived sympathetic neurons. Judging by the lack of protection provided by siRNA R2, this inhibition needs to be near-complete to achieve this effect.

\section{Discussion}

We present here the first conclusive demonstration of the involvement of RAIDD in apoptosis. Although previous studies had shown that RAIDD can associate with caspase 2 and induce death upon overexpression, ${ }^{18,19,22}$ no study so far has directly implicated endogenous RAIDD in apoptosis. These findings are all the more important, because they are applicable to a model of apoptosis in primary neurons, a model that mimics developmental neuronal programmed cell death.

We have previously shown that caspase 2 is a critical mediator of apoptosis in this model. ${ }^{12,13}$ Our present findings reveal another critical piece of this apoptotic mechanism, and indicate that the caspase 2 adaptor RAIDD is also required for this death to occur. We have previously shown that rat RAIDD and caspase 2 , like their human homologues, can interact in a CARD-dependent manner. C2(1-135), a CARD-only truncated version of caspase 2 bound to RAIDD and interfered with RAIDD-induced death. ${ }^{22}$ We show here that expression

Figure 5 RAIDD downregulation promotes survival of serum-deprived PC12 cells. (a) PC12 cells were transfected with pCMS-EGFP and either ctl or R1 or R2 siRNA in 24-well plates. After $36 \mathrm{~h}$, the cells were rinsed extensively in serum-free medium, and then maintained in serum-free or in serum-containing medium. Strip counts of EGFP-positive cells were performed in a blinded manner at baseline and $24 \mathrm{~h}$ later. Results are reported as the percentage of EGFP-positive cells in serum-free versus serum-containing medium for each set of transfections. Coexpression of R1siRNA or R2 siRNA was significantly protective compared to ctl siRNA $\left({ }^{\star} P<0.05,{ }^{*} P=0.01\right)$. Such protective effects were seen in four independent experiments. (b) PC12 cells were transfected with pCMS-EGFP and either scrambled (scr) or R1 siRNA (R1), or R1 siRNA together with human RAIDD (hRAIDD). Serum deprivation and strip-counts of EGFP-positive neurons were performed as above. Results are reported as the percentage of EGFPpositive cells in serum-free versus serum-containing medium for each set of transfections. Coexpression of R1 siRNA was significantly protective $\left({ }^{* \star *} P<0.005\right.$ compared to $\mathrm{scr}$ ), and this effect was completely abrogated by hRAIDD ( $P=0.4$ compared to $s c r)$. Similar results were achieved in two other independent experiments. (c) PC12 cells were transfected and maintained in serum $(+$ serum) or serum-deprived $(-$ serum $)$ as above. They were then fixed and immunostained with an EGFP Ab and labeled with Hoechst. The percentage of EGFP-positive cells that showed evidence of nuclear apoptosis was assessed. Results are representative of three independent experiments. Coexpression of R1siRNA or R2siRNA significantly inhibited apoptosis compared to ctl siRNA $\left({ }^{* \star *} P<0.005\right.$ and $\left.{ }^{*} P<0.05\right)$. (d) $P C 12$ cells were transfected with pCMS-EGFP and either scrambled (scr) or R1 siRNA. After $36 \mathrm{~h}$, the cells were rinsed, and then maintained in complete medium with or without $10 \mu \mathrm{M}$ camptothecin. Strip counts of EGFP-positive cells were performed in a blinded manner at baseline and $24 \mathrm{~h}$ later. Results are reported as the percentage of EGFP-positive cells in cultures treated with camptothecin versus those without treatment. R1siRNA provided no protective effect in two independent experiments 
a

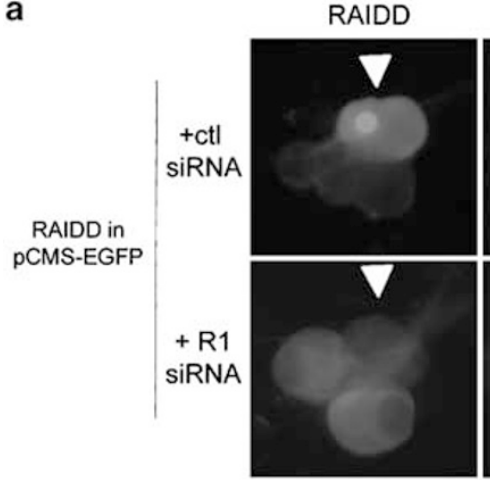

EGFP

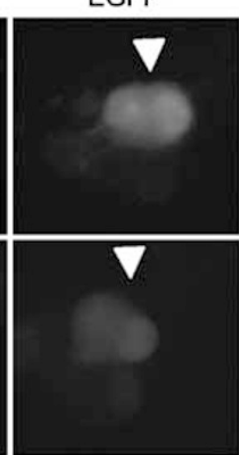

b

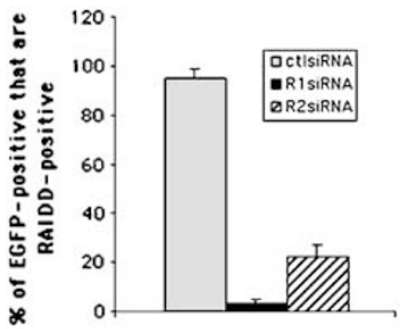

C

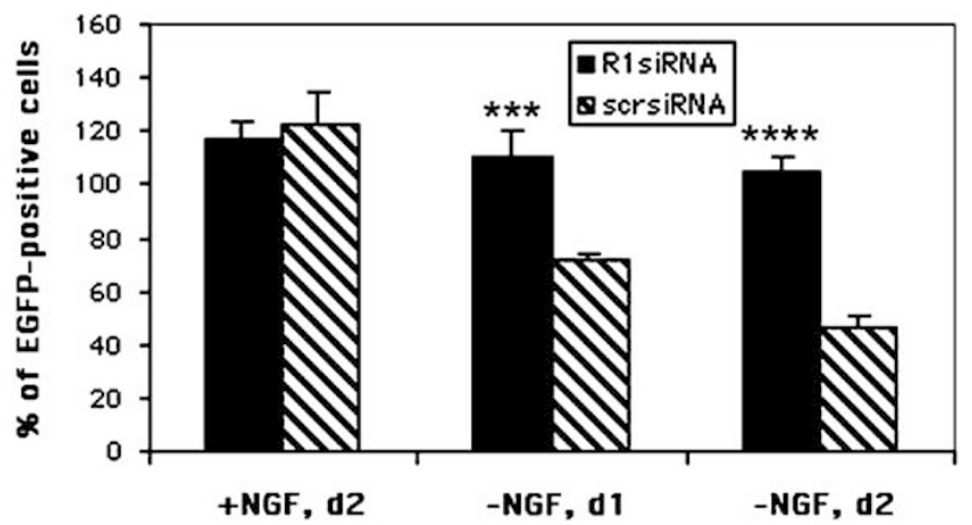

Figure 6 RAIDD downregulation by siRNA promotes survival of NGF-deprived sympathetic neurons. (a) Sympathetic neuron cultures ( 3 days old) were cotransfected with pCMS-EGFP/RAIDD and either control (ctl) or R1 siRNA. After $60 \mathrm{~h}$, cultures were fixed and immunostained for EGFP and RAIDD. Representative examples are shown. Note the high levels of overexpressed RAIDD in an aggregated conformation in an EGFP-expressing neuron in cultures cotransfected with ctl siRNA (arrow head, top panel), and the absence of such overexpression in a neuron cotransfected with R1siRNA (arrow head, bottom panel). Exposure time was 2.5 times longer for the picture of RAIDD immunostaining in the bottom panel compared to the top, to better demonstrate endogenous RAIDD immunostaining, which was also apparently diminished in the one siRNA-transfected neuron (arrow head, bottom panel) compared to the other two neurons in the same visual field. (b) Sympathetic neurons were transfected with pCMS-EGFP/RAIDD and either ctl or R1 or R2 siRNA in 24-well plates. After $60 \mathrm{~h}$, cultures were fixed and immunostained for EGFP and RAIDD. The percentage of EGFP-positive cells that showed intense RAIDD immunostaining was assessed in each setting. Note that only EGFP-positive cells were assessed, and that these represent a minority in the cultures (less than $2 \%$ ). This is representative of two independent experiments. (c) Sympathetic neuron cultures (2 days old) were transfected with pCMS-EGFP and either scrambled (scr) or R1 siRNA in 24-well plates. After $24 \mathrm{~h}$, the cultures were either deprived of NGF (-NGF), or maintained in NGF ( + NGF). Strip counts of EGFP-positive neurons were performed in a blinded manner at baseline (time 0 ) and 1 and 2 days later. At least 75 neurons were counted in each well at time 0 . Results (mean \pm S.E.M., $n=3$ ) are reported as the percentage of EGFP-positive neurons in each condition compared to time 0 . Coexpression of R1siRNA was significantly protective compared to scr siRNA $\left({ }^{* \star *} P<0.005\right.$, and ${ }^{* * * *}<0.001$ at 2 days). Protective effects of R1 siRNA were seen in two other independent experiments

of this construct also inhibits trophic withdrawal-induced apoptosis of PC12 cells and sympathetic neurons. These results suggest that the ability of this truncated protein to interact with RAIDD and inhibit its interaction with endogenous caspase 2 is responsible for its protective effects. The possibility however existed that another caspase 2-interacting molecule, and not RAIDD per se, was involved in apoptotic death in this model. We therefore used an siRNA strategy to downregulate RAIDD expression, and have found that such downregulation results in inhibition of death. siRNAs targeted against two different sites in the RAIDD sequence were both protective in the PC12 cell model, compared to two different ctl siRNAs, and their protective effects correlated with their ability to inhibit RAIDD overexpression. Furthermore, a rescue experiment, in which human RAIDD was reintroduced, showed that this effect was specific for RAIDD. We believe that these results obviate potential concerns for off-target effects of the siRNA technology. To further solidify these findings, we have more recently generated another RNAi against an additional sequence in RAIDD. This siRNA provides comparable suppression of RAIDD expression to $R 1$, and also promotes survival to a similar extent to R1 in PC12 cells. Furthermore, it is also protective against NGF deprivation-induced death in sympathetic neurons (Elena Ribe, Matina Maniati, Leonidas Stefanis and Carol Troy, unpublished observations). These data in conjunction demonstrate that an interaction between caspase 2 and RAIDD is critical for apoptosis in this model.

The exact manner of interaction between caspase 2 and RAIDD and of caspase 2 activation in this model remains unclear. Recently, it was demonstrated that caspase 2 can form high molecular weight (HMW) complexes upon incubation of cell lysates at $37^{\circ} \mathrm{C}$. This complex was formed independently of the caspase 9-related apoptosome, and contained activated caspase $2 .^{26}$ The physiological relevance of this complex remains however unclear, because the conditions utilized may not reflect what occurs in a cellular context following an apoptotic stimulus. A relevant model for 
the activation of caspase 9 , which is highly homologous to caspase 2, has been proposed: Caspase 9 is recruited in a CARD-dependent manner to Apaf-1, resulting in a critical local concentration of caspase 9 , and then its dimerization/oligomerization and subsequent activation. ${ }^{27,28}$ It is possible that, in an analogous manner, in our model RAIDD plays the role of Apaf- 1 in recruiting caspase 2 molecules and thus achieving their activation in an HMW complex through dimerization/autooligomerization. RAIDD self-oligomerization, which we have shown occurs upon its overexpression, $^{22}$ may also play a role in this activation. Support for this idea comes from a recent study that showed that, upon the same conditions of incubation of cell extracts employed by Read et al., ${ }^{26}$ RAIDD forms an HMW complex with caspase 2. ${ }^{29}$ Importantly, this complex also included the protein PIDD, which is related to the signaling pathway of p53. p53 is not thought to be involved in trophic withdrawal-induced neuronal apoptosis (Anderson and Tolkovsky, ${ }^{30}$ but see also Aloyz et $a^{\beta 1}$ ), but PIDD may be constitutively present in our cellular system, and may function independently of the p53 signaling pathway. Also of relevance, in our study, downregulation of RAIDD did not affect death promoted by the DNA-damaging agent camptothecin. Death in this model is known to be p53 dependent. ${ }^{32}$ It is further worth noting that the formation of the caspase 2related HMW complex observed by Tinel and Tschopp ${ }^{29}$ was not linked to apoptosis, and that its potential role in this process remains unclear.

Another question that requires clarification is the cellular compartment in which the RAIDD/caspase 2 interaction and subsequent caspase 2 activation occur. Our initial immunostaining studies had found that caspase 2 mainly localizes to the cytoplasm in PC12 cells and sympathetic neurons. ${ }^{12}$ Subsequent studies however have reported various localizations for caspase 2, most importantly the nucleus, ${ }^{33,34,7}$ the mitochondria $^{35}$ and the Golgi apparatus. ${ }^{36}$ The apparent involvement of caspase 2 as a 'master regulator' of apoptosis, upstream of cytochrome $c$ release, following genotoxic stimuli in certain tumor cell lines has also suggested that its critical action may reside in the nucleus. ${ }^{10}$ We report here that endogenous RAIDD localizes to the cytosol. No appreciable RAIDD localization was found in other cellular compartments in our experiments, in contrast to Shearwin-Whyatt et al., ${ }^{37}$ who found RAIDD localization in both cytoplasm and nucleus of HeLa cells. The caspase 2 pro-form was predominantly processed in the cytosol in our experiments, providing further evidence that the cytosolic compartment is the one in which critical interactions between caspase 2 and RAIDD are likely to take place. The fact that expression of the putative dominant-negative construct C2(1-135) was also exclusively confined to the cytoplasm, many times in the form of discrete puncta, further argues that this is the case, at least in our cellular system.

The finding that RAIDD siRNA does not inhibit death induced by DNA damage is consistent with our previous results, which argued that caspase 2 is involved specifically in the pathway of trophic deprivation-induced neuronal death. ${ }^{12,25}$ Our present data reinforce that idea, and suggest more generally that apoptotic pathways elicited in the same cell may differ depending on the initiating stimulus.
In conclusion, we demonstrate here that RAIDD, likely through a critical interaction with caspase 2 , is involved specifically in trophic deprivation-induced death of PC12 cells and sympathetic neurons. The exact mode of interaction between caspase 2 and RAIDD, the initiating event that promotes such an interaction, other potential binding partners similar to PIDD, and the relationship of these events to other critical points in this apoptotic pathway remain to be elucidated.

\section{Materials and Methods}

\section{Cell culture}

PC12 cells were grown as previously described. ${ }^{12,13}$ Sympathetic neuron cultures were derived from sympathetic ganglia of 1-2-day-old rat pups, and cultured according to the established protocols. ${ }^{12,13}$

\section{SiRNAs}

The ATF5 siRNA that was used as a ctlin some experiments has been reported previously. ${ }^{23}$ We generated two custom-made double-stranded siRNAs (R1: r(GGAAUUUUGACGGAAAACC)d(TT) and R2: r(GGACUG GUUCUUCAGUACC)d(TT) targeted against distinct regions of the sequence of rat RAIDD. ${ }^{22} \mathrm{~A}$ scr siRNA of R1 that did not have any homology in the database was used as an additional ctl. All results that were obtained with R1siRNA and ctl siRNA were verified using the scr siRNA.

\section{Transfection}

The constructs utilized for transfection (RAIDD, FRAIDD, Flag-tagged human RAIDD (FhRAIDD), C2(1-135), C2(1-135M), PAI2M, EGFP) have been previously described. ${ }^{22}$ Transient overexpression of the various genes of interest in PC12 cells or sympathetic neurons was achieved by Lipofectamine-mediated transfection (Lipofectamine 2000, Life Sciences), following the manufacturer's recommendations, with modifications, as described. ${ }^{22}$ siRNAs were transfected in 24-well dishes at a concentration of $25 \mathrm{nM}$ together with $0.3 \mu \mathrm{g}$ of plasmid DNA (either expressing EGFP alone, or together with RAIDD (wild-type rat RAIDD), FRAIDD, or FhRAIDD, (generously provided by Sharad Kumar and Shearwin-Whyatt, Adelaide, Australia). In the case of the pCXN2 vector encoding FhRAIDD, this was coexpressed with the pCMS-EGFP in a $1: 1$ ratio, together with the siRNAs. Concentrations were selected because of preliminary experiments that showed good concordance of fluorescence upon cotransfection of EGFP with fluorescently tagged siRNAs provided by the manufacturer. At $24-36 \mathrm{~h}$ after transfection trophic deprivation was performed.

\section{Trophic deprivation/DNA damage}

PC12 cells were mechanically dissociated from 100 or $35 \mathrm{~mm}$ dishes after five rinses with serum-free RPMI 1640 medium and washed with the same medium 3-4 times by centrifugation and resuspension. PC12 cells were then replated in collagen-coated 24 -well or $35 \mathrm{~mm}$ dishes. ${ }^{12,13}$ For apoptosis induced by DNA damage, cells were exposed to complete medium with or without $10 \mu \mathrm{M}$ camptothecin. ${ }^{24}$

At 3-6 days after plating, sympathetic neurons were rinsed in serum-free medium, and then either re-exposed to medium with NGF 
( + NGF), or deprived of NGF by applying anti-mouse NGF antibody (Ab) (Sigma, $1: 200){ }^{12}$

\section{Assessment of cell death}

To assess survival of transfected trophic factor-deprived PC12 cells and sympathetic neurons, we counted in a blinded manner in a defined area of each well the number of EGFP-positive cells immediately after trophic deprivation or camptothecin application (time 0). We repeated these counts 24 and $48 \mathrm{~h}$ later. We calculated the percentage of EGFP-positive cells present at these time points compared to time 0 . The results in $\mathrm{PC} 12$ cells are reported relative to the percentage of cells that were maintained in serum. We counted at least 100 EGFP-positive PC12 cells or 75 sympathetic neurons per well, in at least three different wells per condition.

To assess cell death of transfected cells, we immunostained the cultures with the appropriate antibodies, and counterstained with Hoechst. We then counted in a blinded manner the percentage of cells expressing the protein of interest that showed apoptotic nuclear morphology. We counted at least 100 immuno-positive PC12 cells or 50 sympathetic neurons per well, in at least three different wells per condition.

Results are reported as mean \pm S.E.M. Statistical comparisons were carried out using Student's $t$-test.

These assessments of survival/death assessments were performed with the siRNA experiments, in which, as noted above, there was a good correlation between EGFP positivity and penetration of siRNAs. This good correlation was further reinforced by the substantial inhibition of RAIDD overexpression, especially with the R1 siRNA (see Results). When we expressed C2(1-135) in a pCMS-EGFP vector, fewer than $50 \%$ of the EGFP-positive cells also showed Myc positivity by immunostaining. Therefore, we have only used the assay of apoptotic death to assess the effects of the expression of this construct on trophic deprivation-induced death.

\section{Immunofluorescence}

PC12 cells or sympathetic neurons plated on collagen-coated dishes or glass coverslips (Carolina Biological Supplies) were fixed in freshly prepared $3.7 \%$ formaldehyde for $25 \mathrm{~min}$ at $4{ }^{\circ} \mathrm{C}$, and then incubated with $10 \%$ normal goat serum (NGS) with $0.4 \%$ Triton $X-100$ to block nonspecific binding, followed by incubation with the primary $A b$ for $1 \mathrm{~h}$ at room temperature. Specific antibodies used were anti-RAIDD (rabbit polyclonal, StressGen AAP-270, $1: 150$ ), anti-Myc (9E10 mouse monoclonal, derived from a mouse hybridoma cell line, 1:1), anti-Flag (mouse monoclonal M2, Sigma, 1:400), anti-EGFP (mouse monoclonal, Santa Cruz, 1:100 or rabbit polyclonal, SYSY, 1:300). Following incubation with fluorescent secondary antibodies (Cy2, 1:100; or Cy3, 1:250, Jackson ImmunoResearch), cells were rinsed in PBS. Hoechst 33342 (Sigma, $1 \mu \mathrm{g} / \mathrm{ml}$ ) was added for $20 \mathrm{~min}$ at RT, followed by rinses in PBS. In certain cases, to ensure specificity of imunofluorescence, the immunizing RAIDD peptide (Stressgen) was preincubated with the RAIDD Ab for $30 \mathrm{~min}$ at a 10-fold excess prior to immunostaining. The cells were then visualized in an inverted microscope (Leica DM IRB) at $\times 40$ or $\times 100$, and representative images were obtained with a digital Spot camera. Identical exposure times were used across conditions, except where noted.

\section{Detergent-soluble lysates}

To generate detergent-soluble lysates, PC12 cells were rinsed with cold PBS, triturated off the dish in PBS, and then pelletted by centrifugation in a microcentrifuge at $4000 \mathrm{rpm}$. Cells were then lysed in a buffer containing
1\% Triton X-100, $25 \mathrm{mM}$ HEPES, $5 \mathrm{mM} \mathrm{MgCl}$, 1 mM EDTA, $1 \mathrm{mM}$ EGTA and protease inhibitors (Complete, Roche), and left on ice for $20 \mathrm{~min}$. Lysates were then centrifuged at $13000 \mathrm{rpm}$ in a microcentrifuge, and the supernatants were used for further analysis.

\section{Subcellular fractionation}

For subcellular fractionation, pelletted $\mathrm{PC} 12$ cells were resuspended in 150-200 $\mu$ of buffer A, containing $300 \mathrm{mM}$ sucrose, $1 \mathrm{mM}$ EDTA, $20 \mathrm{mM}$ MOPS (pH 7.4) and protease inhibitors. Cells were then transferred to a Dounce homogenizer, where they were lysed with 20-30 strokes. Lysates were then centrifuged for $10 \mathrm{~min}$ at $1000 \mathrm{rpms}$ in a microcentrifuge. The pellet represented the crude nuclear fraction, which also contains unbroken cells. This was further processed by resuspension in buffer $\mathrm{B}$ $(0.5 \%$ ethylhexadecyldimethylammonium bromide, $0.28 \%$ glacial acetic acid, $0.5 \%$ Triton X-100, $2 \mathrm{mM} \mathrm{MgCl}_{2}, 2.2 \mathrm{mM} \mathrm{NaCl}, 0.1 \times \mathrm{PBS}$ ), which lyses cells, but leaves nuclear membranes intact. ${ }^{38}$ The nuclear lysate was then centrifuged at $2000 \mathrm{rpms}$ for $10 \mathrm{~min}$, and resuspended in cold PBS. Centrifugation and resuspension in PBS was repeated twice and the final pellet represented the nuclear lysate. The supernatant from the first centrifugation, containing cytosolic and membrane fractions, was centrifuged at $100000 \times g$ for $60 \mathrm{~min}$. The pellet represented the membrane-organelle fraction and the supernatant the S-100 cytosol. All procedures were performed at $4^{\circ} \mathrm{C}$. Protein concentrations were measured using the Bradford assay (BioRad). Lysates were then solublized in sample buffer (SB) plus $\beta$-mercaptoethanol. Specific subcellular compartment markers were used to ensure the validity of this protocol (see Results).

\section{Western immunoblotting}

In total, $50-100 \mu \mathrm{g}$ of protein was separated by SDS-PAGE (12\%, unless specified) and transferred onto nitrocellulose membranes. The membranes were blocked in 5\% non-fat milk for $1 \mathrm{~h}$ at room temperature and probed overnight at $4^{\circ} \mathrm{C}$ with antibodies raised against RAIDD (rabbit polyclonal, 1:500, Stressgen), PARP (mouse monoclonal, 1:5000, Enzyme Systems Products), COX (mouse monoclonal, 1:2500, Molecular Probes), $\beta$-actin (Sigma, $1: 20000)$, Flag (mouse monoclonal, Sigma, $1: 500$ ), or caspase 2 (rabbit polyclonal, $1: 500^{12}$ ). Protein bands were visualized with horseradish peroxidase conjugated secondary antibodies (Pierce) and enhanced chemiluminescence (Pierce). To ctl for protein loading, in certain cases, the membranes were stripped and reprobed with rabbit polyclonal ERK-2 Ab (Santa Cruz, $1: 2000)$.

\section{Acknowledgements}

This work was supported by March of Dimes Grant 1-FY99-318, Burroughs Wellcome Career Award in Biomedical Sciences and a Marie Curie Reintegration Grant (LS), NINDS (LAG and CMT) and MDA (CMT).

\section{References}

1. Rideout HJ and Stefanis $L$ (2001) Caspase inhibition: a potential therapeutic strategy in neurological diseases. Histol. Histopathol. 16: 895-908

2. Degterev A, Boyce M and Yuan J (2003) A decade of caspases. Oncogene 22: 8543-8567

3. Lamkanfi M, Declercq W, Kalai M, Saelens X and Vandenabeele P (2002) Alice in caspase land. A phylogenetic analysis of caspases from worm to man. Cell Death Differ. 9: 358-361 
4. Troy CM and Shelanski ML (2003) Caspase-2 redux. Cell Death Differ. 10: 101-107

5. Kumar S and Vaux DL (2002) Apoptosis. A cinderella caspase takes center stage. Science 297: 1290-1291

6. Guo Y, Srinivasula SM, Druilhe A, Fernandes-Alnemri T and Alnemri ES (2002) Caspase-2 induces apoptosis by releasing proapoptotic proteins from mitochondria. J. Biol. Chem. 277: 13430-13437

7. Paroni G, Henderson C, Schneider C and Brancolini C (2001) Caspase-2induced apoptosis is dependent on caspase-9, but its processing during UV- or tumor necrosis factor-dependent cell death requires caspase-3. J. Biol. Chem. 276: 21907-21915

8. Paroni G, Henderson C, Schneider C and Brancolini C (2002) Caspase-2 can trigger cytochrome $C$ release and apoptosis from the nucleus. J. Biol. Chem. 277: 15147-15161

9. Robertson JD, Enoksson M, Suomela M, Zhivotovsky B and Orrenius S (2002) Caspase-2 acts upstream of mitochondria to promote cytochrome $c$ release during etoposide-induced apoptosis. J. Biol. Chem. 277: 29803-29809

10. Lassus P, Opitz-Araya $X$ and Lazebnik $Y$ (2002) Requirement for caspase-2 in stress-induced apoptosis before mitochondrial permeabilization. Science 297: 1352-1354

11. Baliga B and Kumar S (2003) Apaf-1/cytochrome $c$ apoptosome: an essential initiator of caspase activation or just a sideshow? Cell Death Differ. 10: 16-18

12. Troy CM, Stefanis L, Greene LA and Shelanski ML (1997) Nedd2 is required for apoptosis after trophic factor withdrawal, but not superoxide dismutase (SOD1) down-regulation, in sympathetic neurons and PC12 cells. J. Neurosci. 17: 1911-1918

13. Stefanis L, Troy CM, Qi H, Shelanski ML and Greene LA (1998) Caspase-2 (Nedd-2) processing and death of trophic factor-deprived PC12 cells and sympathetic neurons occur independently of caspase-3 (CPP32)-like activity. J. Neurosci. 18: 9204-9215

14. Bergeron L, Perez Gl, Macdonald G, Shi L, Sun Y, Jurisicova A, Varmuza $S$, Latham KE, Flaws JA, Salter JC, Hara H, Moskowitz MA, Li E, Greenberg A, Tilly $\mathrm{JL}$ and Yuan $\mathrm{J}$ (1998) Defects in regulation of apoptosis in caspase-2deficient mice. Genes Dev. 12: 1304-1314

15. O'Reilly LA, Ekert P, Harvey N, Marsden V, Cullen L, Vaux DL, Hacker G Magnusson C, Pakusch M, Cecconi F, Kuida K, Strasser A, Huang DC and Kumar S (2002) Caspase-2 is not required for thymocyte or neuronal apoptosis even though cleavage of caspase-2 is dependent on both Apaf- 1 and caspase9. Cell Death Differ. 9: 832-841

16. Troy CM, Rabacchi SA, Hohl JB, Angelastro JM, Greene LA and Shelanski ML (2001) Death in the balance: alternative participation of the caspase-2 and -9 pathways in neuronal death induced by nerve growth factor deprivation. J. Neurosci. 21: 5007-5016

17. Yu LY, Jokitalo E, Sun YF, Mehlen P, Lindholm D, Saarma M and Arumae $U$ (2003) GDNF-deprived sympathetic neurons die via a novel nonmitochondrial pathway. J. Cell Biol. 163: 987-997

18. Duan H and Dixit VM (1997) RAIDD is a new 'death' adaptor molecule. Nature 385: 86-89

19. Ahmad M, Srinivasula SM, Wang L, Talanian RV, Litwack G, FernandesAlnemri T and Alnemri ES (1997) CRADD, a novel human apoptotic adaptor molecule for caspase-2, and FasL/tumor necrosis factor receptor-interacting protein RIP. Cancer Res. 57: 615-619

20. Koseki T, Inohara N, Chen S and Nunez G (1999) ARC, an inhibitor of apoptosis expressed in skeletal muscle and heart that interacts selectively with caspases. Proc. Natl. Acad. Sci. USA 95: 5156-5160
21. Dowds TA and Sabban EL (2001) Endogenous and exogenous ARC in serum withdrawal mediated PC12 cell apoptosis: a new pro-apoptotic role for ARC. Cell Death Differ. 8: 640-648

22. Jabado O, Wang $Q$, Rideout HJ, Yeasmin M, Guo KX, Vekrellis K, Papantonis $S$, Angelastro JM, Troy CM and Stefanis L (2004) RAIDD aggregation facilitates apoptotic death of PC12 cells and sympathetic neurons. Cell Death Differ. 11: $618-630$

23. Angelastro JM, Ignatova TN, Kukekov VG, Steindler DA, Stengren GB, Mendelsohn C and Greene LA (2003) Regulated expression of ATF5 is required for the progression of neural progenitor cells to neurons. J. Neurosci. 23: $4590-4600$

24. Park DS, Morris EJ, Greene LA and Geller HM (1997) G1/S cell cycle blockers and inhibitors of cyclin-dependent kinases suppress camptothecin-induced neuronal apoptosis. J. Neurosci. 17: 1256-1270

25. Park DS, Morris EJ, Stefanis L, Troy CM, Shelanski ML, Geller HM and Greene LA (1998) Multiple pathways of neuronal death induced by DNA-damaging agents, NGF deprivation, and oxidative stress. J. Neurosci. 18: 830-840

26. Read SH, Baliga BC, Ekert PG, Vaux DL and Kumar S (2003) A novel Apaf-1independent putative caspase-2 activation complex. J. Cell Biol. 159: 739-745

27. Srinivasula SM, Ahmad M, Fernandes-Alnemri T and Alnemri ES (1998) Autoactivation of procaspase- 9 by Apaf-1-mediated oligomerization. Mol. Cell 1: 949-957

28. Boatright KM, Renatus M, Scott FL, Sperandio S, Shin H, Pedersen IM, Ricci JE, Edris WA, Sutherlin DP, Green DR and Salvesen GS (2003) A unified model for apical caspase activation. Mol. Cell 11: 529-541

29. Tinel A and Tschopp J (2004) The PIDDosome, a protein complex implicated in activation of caspase-2 in response to genotoxic stress. Science 304: 843-846

30. Anderson CN and Tolkovsky AM (1999) A role for MAPK/ERK in sympathetic neuron survival: protection against a p53-dependent, JNK-independent induction of apoptosis by cytosine arabinoside. J. Neurosci. 19: 664-673

31. Aloyz RS, Bamji SX, Pozniak CD, Toma JG, Atwal J, Kaplan DR and Miller FD (1998) p53 is essential for developmental neuron death as regulated by the TrkA and p75 neurotrophin receptors. J. Cell Biol. 143: 1691-1703

32. Morris EJ, Keramaris E, Rideout HJ, Slack RS, Dyson NJ, Stefanis L and Park DS (2001) Cyclin-dependent kinases and P53 pathways are activated independently and mediate Bax activation in neurons after DNA damage. J. Neurosci. 21: 5017-5026

33. Zhivotovsky B, Samali A, Gahm A and Orrenius S (1999) Caspases: their intracellular localization and translocation during apoptosis. Cell Death Differ. 6: 644-651

34. Colussi PA, Harvey NL and Kumar S (1998) Prodomain-dependent nuclear localization of the caspase-2 (Nedd2) precursor. A novel function for a caspase prodomain. J. Biol. Chem. 273: 24535-24542

35. Susin SA, Lorenzo HK, Zamzami N, Marzo I, Brenner C, Larochette N, Prevost MC, Alzari PM and Kroemer G (1999) Mitochondrial release of caspase-2 and 9 during the apoptotic process. J. Exp. Med. 189: 381-394

36. Mancini M, Machamer CE, Roy S, Nicholson DW, Thornberry NA, CasciolaRosen LA and Rosen A (2000) Caspase-2 is localized at the Golgi complex and cleaves golgin-160 during apoptosis. J. Cell Biol. 149: 603-612

37. Shearwin-Whyatt LM, Harvey NL and Kumar S (2000) Subcellular localization and CARD-dependent oligomerization of the death adaptor RAIDD. Cell Death Differ. 7: 155-165

38. Rideout HJ, Wang Q, Park DS and Stefanis L (2003) Cyclin-dependent kinase activity is required for apoptotic death but not inclusion formation in cortical neurons after proteasomal inhibition. J. Neurosci. 23: 1237-1245 